\title{
Increased Risk of Revision, Reoperation, and Implant Constraint in TKA After Multiligament Knee Surgery
}

\author{
Steven I. Pancio MD, Paul L. Sousa MD, Aaron J. Krych MD, \\ Matthew P. Abdel MD, Bruce A. Levy MD, Diane L. Dahm MD, \\ Michael J. Stuart MD
}

Received: 21 September 2016/Accepted: 4 January 2017/Published online: 13 January 2017

(C) The Association of Bone and Joint Surgeons \& 2017

\begin{abstract}
Background The risk of major complications and revision arthroplasty after TKA in patients who previously underwent multiligament knee surgery have been poorly characterized.

Questions/purposes Is multiligament knee surgery before TKA associated with (1) worse implant survival, (2) increased use of TKA design constraint, (3) a greater risk for major complications, and (4) poorer scores for pain and
\end{abstract}

One or more of the authors (MJS, BAL, AJK) is a paid consultant for Arthrex (Naples, FL, USA).

One or more of the authors certifies that he or she (BAL, DLD, MJS, AJK) or a member of his or her immediate family, has or may receive payments or benefits, during the study period, an amount of USD 10,000-100,000 from Arthrex (Naples, FL, USA), and an amount of USD 10,000-100,000 from Tenex Health (Lake Forest, CA, USA). One of the authors (BAL) receives research support from Stryker (Mahwah, NJ, USA), Biomet (Warsaw, IN, USA), Smith and Nephew (Andover, MA, USA), and Arthrex (Naples, FL, USA). One of the authors (MJS) receives research support from Stryker (Mahwah, NJ, USA) and the USA Hockey Foundation (Colorado Springs, CO, USA).

One of the authors (AJK) receives research support from the Arthritis Foundation (Atlanta, GA, USA), Histogenics (Waltham, MA, USA), and Ceterix (Fremont, CA, USA).

All ICMJE Conflict of Interest Forms for authors and Clinical Orthopaedics and Related Research ${ }^{\circledR}$ editors and board members are on file with the publication and can be viewed on request.

Each author certifies that his or her institution approved the human protocol for this investigation that all investigations were conducted in conformity with ethical principles of research, and that informed consent for participation in the study was obtained.

S. I. Pancio, P. L. Sousa, A. J. Krych, M. P. Abdel, B. A. Levy, D. L. Dahm, M. J. Stuart ( $\varangle)$

Department of Orthopedic Surgery, Mayo Clinic, 200 First Street

SW, Rochester, MN 55905, USA

e-mail: Stuart.Michael@mayo.edu function compared with similar patients receiving TKA for primary osteoarthritis?

Methods Fifty-nine TKAs were performed at our institution between 1985 and 2014 in 59 patients (36 men, 23 women; mean age, 53 years) with a history of previous multiligament knee surgery ( $\geq$ two ligaments). Of those, we had followup for $39(66 \%), 18(31 \%)$, and six (10\%) patients at 5,10, and 15 years, respectively; mean followup was 5.4 years (range, 1-25 years). A two-to-one matched control group consisting of patients undergoing primary TKA for the diagnosis of osteoarthritis was selected for comparison. Patients were matched based on gender, age at primary TKA (within 5 years), and date of the TKA (within 5 years). Medical records were reviewed for survivorship, TKA design, complications (reoperation, revision, infection, manipulation under anesthesia, and periprosthetic joint infection), TKA design, and clinical outcomes (Knee Society Scores [KSS], Knee Society Function Score [KSSF]).

Results The overall 15-year revision-free survival in patients with prior multiligament knee surgery was decreased in comparison to the matched controls (42\% [95\% CI, 16\%-73\%] vs 94\% [95\% CI, 81\%-99\%]; p < 0.001). Varus-valgus constraint implant design was used for more patients in the multiligament cohort at index TKA than in the matched control group (9/59 [15\%] vs $0 / 110$ [0\%], respectively; odds ratio [OR], 45; 95\% CI, 3-781; $\mathrm{p}=0.009)$. Patients with a history of multiligament knee surgery also were at increased risk of reoperation for any cause (14/59 [24\%] vs 7/118 [6\%]; OR, 5; 95\% CI, 2-14; $\mathrm{p}=0.001$ ). With the numbers available, there was no difference in the frequency of manipulation under anesthesia after TKA (10\% [6/59] versus 3\% [4/118]; $p=0.08) \mathrm{A}$ higher proportion of patients in the multiligament cohort had infections develop compared with the matched controls 
$(4 / 59$ [7\%] vs $1 / 118[<1 \%)]$, respectively; $\mathrm{p}=0.04)$. There was no difference in the KSS improvement after TKA between the multiligament group and the control group (34 \pm 18 vs $28 \pm 15 ; \mathrm{p}=0.088)$. The final KSS and KSS-F scores likewise showed no difference between those groups $(88 \pm 13$ vs $85 \pm 10 ; \mathrm{p}=0.232)(85 \pm 17$ vs $84 \pm 14 ; \mathrm{p}=$ $0.75)$.

Conclusions A history of multiligament surgery is associated with lower long-term survivorship, higher use of constrained TKA designs, and higher risk of major complications, including reoperation and infection. Further research is necessary to determine if a particular multiligamentous surgical technique can prevent posttraumatic arthritis and TKA complications.

Level of Evidence Level III, therapeutic study.

\section{Introduction}

Multiligament knee injuries are rare but severe traumatic events that are challenging for the patient and the surgeon. Neurovascular compromise and joint stability are of primary initial concern $[11,22-24,40,51-53]$, but the development of posttraumatic arthritis can lead to longterm disability [9, 55, 56]. Ligament disruption often is associated with meniscus, articular cartilage, and subchondral bone injuries. The initial damage in combination with altered knee kinematics can result in accelerated degenerative changes $[3,4,6,10,13,15,16,25-27,33$, 35, 37, 39, 45, 55]. Kaeding et al. [15] identified frequent meniscal tears and chondral injuries in their patients at the time of multiligament knee reconstruction along with increased meniscal tears and joint degeneration with time. Krych et al. [17] reported chondral or meniscus injuries in more than $75 \%$ of patients undergoing multiligament reconstruction after knee dislocation. As a result, patients with a history of knee ligament reconstruction often undergo TKA at a younger age [4]. Leroux et al. [21] showed that previous ACL reconstruction (singleligament knee injury) was associated with a sevenfold increase in TKA when compared with the general population. Fanelli et al. [12] reported a 7\% incidence of TKA at 10-year followup in patients treated surgically for knee dislocation.

Arthroplasty after multiligamentous knee injury has increased surgical complexity owing to altered joint kinematics, retained hardware, loss of bone stock, and scarring from prior surgical intervention resulting in a potential increased risk of TKA failure and major postoperative complications. Whether these issues decrease implant survival or increase the risk of complications after TKA is not known.
We therefore performed a retrospective case-control study in which we asked: Is multiligament knee surgery before TKA associated with (1) worse implant survival, (2) increased use of design constraint, (3) a greater risk for major complications, and (4) poorer scores for pain and function compared with scores for similar patients receiving TKA for primary osteoarthritis?

\section{Patients and Methods}

All patients undergoing TKA between 1985 and 2014 with a history of reconstruction or repair of two or more knee ligaments were identified from our institution's arthroplasty registry after institutional review board approval. Three hundred eighty-nine (2\%) of the 17,482 patients undergoing primary TKA in our registry were found to have a history of any knee ligament surgery. Three hundred thirty patients with previous single-knee ligament surgery were excluded from the study. The remaining $59(0.3 \%)$ patients comprised the multiligament study group. The cohort included 36 men (61\%) and 23 women $(39 \%)$ with a mean age at the time of arthroplasty of 53 years (range, 36-79 years), and a mean BMI of $31 \mathrm{~kg}$ / $\mathrm{m}^{2}$ (range, $21-49 \mathrm{~kg} / \mathrm{m}^{2}$ ) (Table 1). A majority of these patients underwent surgery on the ACL and medial collateral ligament (MCL), ACL, posterior cruciate ligament (PCL), and MCL, or the ACL and PCL structures (Table 2). Of those, we had followup for 39 (66\%), 18 $(31 \%)$, and six (10\%) patients at 5, 10, and 15 years, respectively; mean followup was 5 years (range, 1-25 years). We paired patients in the multiligament surgery cohort to a matched osteoarthritis control group using the same arthroplasty registry database. Patients undergoing primary TKA for osteoarthritis were matched in a two-toone fashion (control: cohort) to the study patients according to: age at TKA (within 5 years), gender, and date of the primary TKA (within 5 years). Patients with prior knee ligament surgery were excluded from the control cohort. A total of 436 potential matches were identified using our institutional joint registry database based on these criteria. Of these, 94 (22\%) were lost to followup before 2 years and were excluded. During the matching process, patients with 2 or more years followup and closest chronologic age to the study patient were selected in the event of multiple potential matches. We identified a control group of 118 patients ( 72 males, 46 females) with a mean age at the time of TKA of 54 years (range, 34-81 years) and BMI of $33 \mathrm{~kg}$ / $\mathrm{m}^{2}$ (range, $18-58 \mathrm{~kg} / \mathrm{m}^{2}$ ) (Table 1). Of those, followup was available for $81(69 \%), 58(49 \%)$, and 17 (14\%) patients at 5,10 , and 15 years, respectively; the mean followup in the control group was 5 years (range, 2-22 years) (Table 1). 
Patient gender, age at the time of TKA, date of primary or revision TKA, BMI, incidence of reoperation (manipulation, irrigation and débridement, revision surgery), level of TKA design constraint (PCL-substituting, varus-valgus constrained implants, hinged implants), incidence of infection and pre- and postoperative Knee Society Score (KSS) and Knee Society Function Score (KSS-F) were recorded. Individual patient registry data were crosschecked with the medical record to ensure validity. Operative reports and clinical notes were reviewed to determine the outcome parameters. Duration of followup was determined based on the most recent clinical appointment and/or availability of the TKA analysis questionnaire.

Table 1. Patient demographics

\begin{tabular}{llll}
\hline Demographic & $\begin{array}{l}\text { Multiligament } \\
\text { cohort }\end{array}$ & $\begin{array}{l}\text { Control } \\
\text { cohort }\end{array}$ & p Value \\
\hline Number & 59 & 118 & - \\
Mean age (years) & $53(36-79)$ & $54(34-81)$ & 0.57 \\
Followup (years) & $5.4(1-25)$ & $5.4(2-25)$ & 0.97 \\
Mean BMI $\left(\mathrm{kg} / \mathrm{m}^{2}\right)$ & $31(21-49)$ & $33(18-58)$ & 0.053 \\
Male:female & $36: 23$ & $72: 46$ & 1.0 \\
\hline
\end{tabular}

Ranges in parentheses.

Table 2. Multiligament surgery

\begin{tabular}{lc}
\hline Ligaments & Number \\
\hline ACL/MCL & 32 \\
ACL/PCL/MCL & 10 \\
ACL/PCL & 8 \\
PCL/MCL & 3 \\
ACL/LCL & 3 \\
ACL/PLC & 2 \\
PCL/PLC & 1 \\
\hline
\end{tabular}

$\mathrm{ACL}=$ anterior cruciate ligament $\mathrm{MCL}=$ medial cruciate ligament; $\mathrm{PCL}=$ posterior cruciate ligament.
Increased TKA design constraint (varus-valgus constrained) was used at the discretion of the primary surgeon for patients with medial and lateral collateral ligament deficiency or irreconcilable flexion-extension mismatch not addressable with standard PCL-substituting implants Patients were evaluated at either a return appointment or by telephone for postoperative ROM. Manipulation under anesthesia was performed if they were found to have less than $90^{\circ}$ knee flexion between 6 and 10 weeks postoperative.

Pre- and postoperative KSS pain and function scores were longitudinally maintained and retrospectively reviewed. Preoperative KSS and KSS-F were available for 34 patients $(58 \%)$ in the multiligament cohort and 60 patients $(51 \%)$ in the matched cohort. Postoperative KSS and KSS-F were available for 53 patients $(95 \%)$ in the multiligament cohort and 105 patients $(89 \%)$ in the matched control group (Table 3). The most recent postoperative KSS and KSS-F available for each patient were used when available regardless of the revision or reoperation history. The multiligamentous repair or reconstructions were performed at various institutions including our institution. Multiligament surgeries that were done at our institution were performed by fellowship trained sports medicine physicians. All TKAs in the multiligament and matched control cohorts were performed by various surgeons at our institution using numerous TKA prosthesis designs. All surgeons were either fellowship trained in adult reconstruction or perform more than 60 TKAs per year. Our primary study comparison was 15 -year cumulative revision-free TKA survivorship.

Statistical Analysis

All outcomes were analyzed using applicable summary statistics. Baseline variables and outcomes were compared using chi-square and Fisher's exact tests (categorical variables), and two-sample paired t-tests (continuous

Table 3. KSS/KSS-F outcomes

\begin{tabular}{llll}
\hline Variable & Multiligament & Control & $\mathrm{p}$ Value \\
\hline KSS preoperative - number & $34 / 59(58 \%)$ & $60 / 118(51 \%)$ \\
KSS-F preoperative - number & $34 / 59(58 \%)$ & $60 / 118(51 \%)$ & - \\
KSS postoperative - number & $53 / 59(90 \%)$ & $105 / 118(89 \%)$ \\
KSS-F postoperative - number & $53 / 59(90 \%)$ & $105 / 118(89 \%)$ & $57(15-87)[54-60]$ \\
Mean KSS preoperative (range)[95\% CI] & $53(20-75)[48-57]$ & $63(30-100)[59-67]$ & - \\
Mean KSS-F preoperative (range)[95\% CI] & $69(40-100)[63-64]$ & $85(67-100)[83-87]$ & $84(50-100)[81-87]$ \\
Mean KSS postoperative (range)[95\% CI] & $88(57-100)[84-92]$ & $28 \pm 15$ & 0.80 \\
Mean KSS-F postoperative (range)[95\% CI] & $85(60-100)[79-91]$ & $31 \pm 30$ & 0.17 \\
Delta KSS & $34 \pm 18$ & 0.75 \\
Delta KSS-F & $21 \pm 18$ & 0.088 \\
\hline
\end{tabular}

KSS = Knee Society Score; KSS-F = Knee Society Function Score. 
variables) as applicable. Odds ratios (OR) with 95\% CIs were used for statistical comparison. Kaplan-Meier survivorship analysis was performed with revision arthroplasty as the endpoint; this was considered suitable because only three patients $(5 \%)$ were known to have died during the surveillance period. Reoperation was defined as any additional surgical procedure including revision arthroplasty, irrigation and débridement, and manipulation under anesthesia. Revision arthroplasty was defined as revision of any or all TKA components (femur, tibia, both) or revision of polyethylene component for reasons other than infection (ie, instability). All statistical tests were two-sided and the threshold for statistical significance was set at an $\alpha$ less than 0.05 .

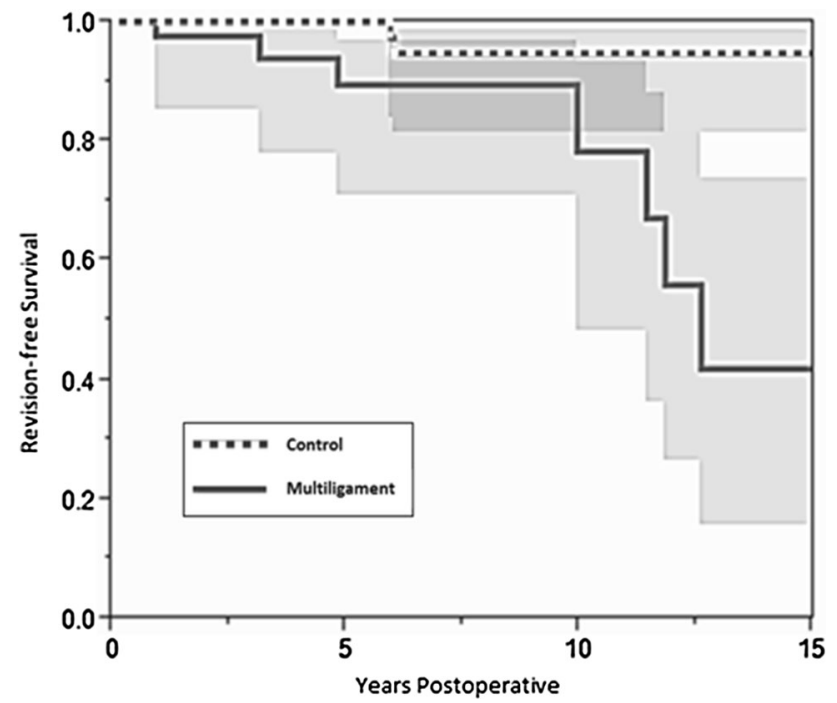

Fig. 1 The Kaplan-Meier survival curve shows there is a decrease in revision-free implant survival in patients with a history of multiligament knee surgery at 15 years after TKA compared with those with a primary diagnosis of knee osteoarthritis $(p<0.05)$.

\section{Results}

Survival

Overall, 15 year revision-free implant survival was decreased in the multiligament cohort $(42 \%$; 95\% CI, $16 \%-73 \%$ ) compared with the matched control cohort (94\%; 95\% CI, 81\%-99\%; p < 0.001). There was also a lower 5-year revision free survival between the multiligament and control cohorts $(89 \%$ [95\% CI, 71\%-97\%] vs $100 \%$ [95\% CI, not applicable], respectively; $\mathrm{p}<0.001$ ). There was no difference in 10-year survival between the two study groups (78\% [95\% CI, 48\%-93\%] vs 94\% [95\% CI, 81\%-99\%]; p = 0.37) (Fig. 1). Eight revision arthroplasties in the multiligament group were performed at an average of 7.5 years after primary TKA $(8 / 59 ; 14 \%$; range, 2-18 years). In total, five patients had revision surgery because of instability, two for infection, and one for aseptic loosening. Two revision procedures were performed in the matched control group, both for aseptic loosening (2/118; $2 \%$ ). There was a higher rate of revision arthroplasty in the multiligament cohort compared with the matched control group (OR, 9; 95\% CI, 2-44; p = 0.006) (Table 4).

\section{Constraint}

Fifteen percent (9/59) of the patients in the multiligament cohort received a varus-valgus constraint prosthesis at the index arthroplasty; this was higher than the control group (0/118; 0\%; OR, 45; 95\% CI, 3-781; p =0.009). A varusvalgus constraint prosthesis was used for only one patient in the matched control group at revision arthroplasty $(1 / 11$; $1 \%$ ). Ultimately, $25 \%$ (15/59) of patients in the multiligament cohort received a TKA with an increased design

Table 4. Clinical outcomes

\begin{tabular}{|c|c|c|c|c|}
\hline Outcome & Multiligament & Control & $\begin{array}{l}\text { Odds ratio } \\
(95 \% \mathrm{CI})\end{array}$ & $\mathrm{p}$ Value \\
\hline Revision & $8 / 59(14 \%)$ & $2 / 118(2 \%)$ & $9(2-44)$ & $\mathrm{p}=0.006$ \\
\hline \multicolumn{5}{|l|}{ Increased constraint* } \\
\hline Total & $15 / 59(25 \%)$ & $1 / 118(1 \%)$ & $39(5-311)$ & $\mathrm{p}<0.001$ \\
\hline At primary TKA & $9 / 59(15 \%)$ & $0 / 118(0 \%)$ & $45(3-781)$ & $\mathrm{p}=0.009$ \\
\hline Reoperation & $14 / 59(24 \%)$ & $7 / 118(6 \%)$ & $4.9(2-14)$ & $\mathrm{p}=0.001$ \\
\hline Infection & $4 / 59(7 \%)$ & $1 / 118(<1 \%)$ & $9(1-78)$ & $\mathrm{p}=0.047$ \\
\hline Two-stage revision & $\mathrm{n}=1$ & $\mathrm{n}=0$ & & \\
\hline Polyethylene exchange & $\mathrm{n}=3$ & $\mathrm{n}=1$ & & \\
\hline Manipulation & $6 / 59(10 \%)$ & $4 / 118(3 \%)$ & $3(0.9-12)$ & $\mathrm{p}=0.08$ \\
\hline
\end{tabular}

* Varus-valgus constraint or hinged prosthesis. 
constraint (varus-valgus constraint or hinged prosthesis) at either primary or revision arthroplasty (Table 4).

\section{Complications}

Patients undergoing TKA after multiligament surgery were at increased risk of major complications; including, reoperation and infection. Fourteen patients $(14 / 59 ; 24 \%)$ in the multiligament cohort underwent reoperation for any cause; eight patients $(5 / 59 ; 14 \%)$ underwent revision TKA and six $(6 / 59 ; 10 \%)$ patients underwent manipulation for stiffness or arthrofibrosis. In comparison to the matched cohort, patients with prior multiligamentous surgery had an increased risk of reoperation (14/59 [24\%] vs 7/118 [6\%]; OR, 5; 95\% CI, 2-14; p = 0.001) (Table 4). Four patients were treated for infection in the multiligament cohort (4/ 59; 7\%). A two-stage revision arthroplasty was performed in three patients (two after primary TKA, one after previous revision TKA). One patient was treated with irrigation and débridement with polyethylene exchange for acute infection. In comparison, only one patient in the matched cohort was treated for infection $(1 / 118 ;<1 \%)$. This patient was treated with irrigation and débridement and polyethylene exchange for acute infection. There was an increased risk of infection in the multiligament cohort compared with the control group (4/59 [7\%] vs $1 / 118[<$ 1\%]; OR, 9; 95\% CI, 1-78; p = 0.047) (Table 4).

Ten percent of the patients in the multiligament cohort (6/59) underwent knee manipulation for postoperative arthrofibrosis or stiffness. Compared with four patients in the matched control group there was no difference in rate of manipulation under anesthesia between the two groups (6/59 (10\%) vs 4/118 (3\%); OR, 3; 95\% CI, 0.9-12; p = 0.079) (Table 4).

\section{Pain and Function Scores}

Pre- and postoperative mean KSS and mean KSS-F were not different with the numbers available between the multiligament group and matched controls (preoperative: mean KSS: cohort 53, control 57 [p $=0.80]$, mean KSS-F: multiligament 69 , control 63 [p $=0.09]$, postoperative: mean KSS: multiligament 88 , control 85 [p $=0.17$ ], mean KSS-F: multiligament 85 , control 84 [p = 0.75]) There was no difference with the numbers available in mean KSS improvement at most recent followup between the multiligament and control cohorts $(34 \pm 18$ versus $28 \pm 15 ; \mathrm{p}=$ 0.088). There also was no difference in mean KSS-F improvement at most recent followup between the multiligament cohort and control groups $(21 \pm 18$ versus $31 \pm$ $30 ; \mathrm{p}=0.098)$ (Table 3).

\section{Discussion}

Knee dislocation and multiligament knee trauma are events that predispose patients to the development of posttraumatic arthritis and the potential need for TKA. Arthroplasty in such patients has increased surgical complexity owing to compromised soft tissues, retained hardware, and distorted anatomy or kinematics (Fig. 2). Consequently, this requires thoughtful decision making at the time of knee arthroplasty that is distinct from TKA for degenerative osteoarthritis. The goal of our study was to retrospectively evaluate implant survival, complication rate, and long term-outcomes of knee arthroplasty in this high-risk population. Our results showed that TKA in patients with a prior history of multiligament surgery has decreased long-term implant survival, increased the risk of revision or reoperation, resulted in more-frequent need for a constrained prosthetic design (varus-valgus constraint), and increased the rate of infection compared with results for patients undergoing primary knee arthroplasty for osteoarthritis.
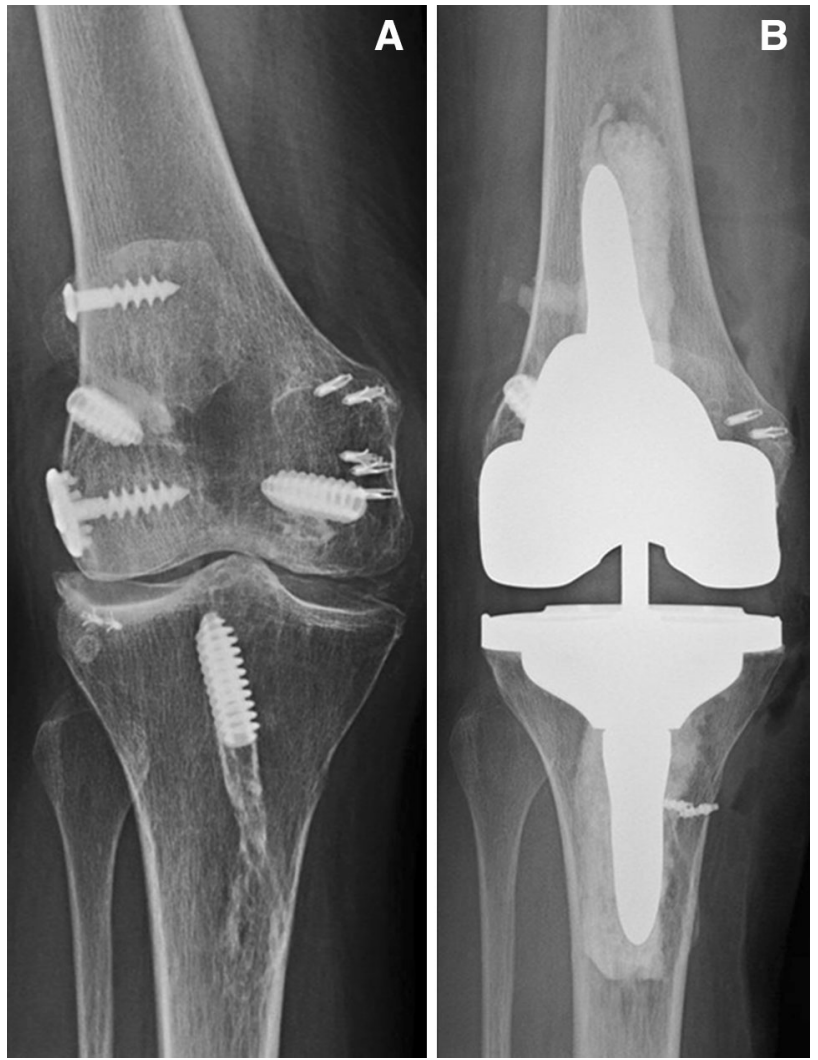

Fig. 2A-B (A) An AP radiograph obtained before arthroplasty shows posttraumatic arthrosis with retained hardware after multiligament surgery. (B) A postoperative AP radiograph shows the cemented TKA with stemmed femur and tibia components and varus-valgus constraint. 
The results of our study should be evaluated in light of several limitations. Patient data were longitudinally collected, but the information was retrospectively reviewed only for patients who had available data at individual times (5, 10, or 15 years). This may introduce transfer bias as patients lost to followup may have a more-negative outcome. However, the percentages of patients available at 5 , 10 , and 15 years in the multiligament and control cohorts were similar, which lessens this phenomenon in a comparison study. Preoperative KSS and KSS-F scores were available for only $58 \%$ of the multiligament patients and $51 \%$ of the controls; furthermore, attrition of KSS and KSS-F score reporting in the study patients as a function of time limited our ability to formulate concrete estimates of patient-reported outcomes after TKA. In addition, the TKAs in our analysis were performed by multiple surgeons using numerous prosthesis designs. Selection bias can result from variations in surgical technique and patient care decision-making affecting patient outcomes. Patient matching for additional factors, such as BMI, ROM, and joint deformity would be ideal, but the relatively small number of available patients precluded matching of all relevant variables. We did match patients to controls on the basis of age, gender, and date of the TKA in an attempt to account for some of the potential variability. For example, patients were matched according to TKA date (within 5 years) in an attempt to limit bias related to procedural technique and evolution of TKA implant design. In addition, all TKAs were performed by high-volume arthroplasty surgeons at our institution, thus reducing technical bias based on surgeon variation. The multiligament repair and reconstruction procedures were performed at multiple institutions including our institution, using diverse surgical techniques which may confound our results; this fact certainly limited the amount of information we had available regarding the index ligamentreconstruction procedures. Finally, many providers were involved in data collection spanning the course of several decades. Assessment bias could artificially overestimate treatment effect as recording the KSS and KSS-F was performed by providers who were directly involved in patient care.

TKA after multiligament knee reconstruction surgery results in poorer cumulative long-term revision-free implant survival compared with TKA for primary knee arthrosis (42\% and 94\%, respectively) (Fig. 1). We also observed a difference in revision-free survival at 5-years after TKA, coinciding with early failures in the study group (two with infections, one with instability). Existing studies that examined survival of TKA after previous knee surgery are limited and conflicting [32, 42, 43, 46]. This is the first study, to our knowledge, that directly examines TKA after multiligament surgery. The explanation for our findings is multifaceted, but inferior survival in the study cohort was largely influenced by instability (5/8) and infection (2/8). Le et al. [20] showed infection and instability to be the most-common causes for late failure after primary TKA. Four of the eight patients in the multiligament cohort who underwent revision arthroplasty did so between 10 and 15 years after the index arthroplasty. More importantly, three of these four revisions were performed for knee instability. The nature of these late failures is noteworthy given the inherent risk of instability after multiligament knee injury and surgery. This young, active patient population with a mean age of 53 years at the time of arthroplasty has a notable risk for TKA failure 10 years after primary arthroplasty.

We found that patients with prior multiligament knee surgery were more likely to receive a varus-valgus constrained device at their index TKA than patients undergoing TKA for osteoarthritis. This finding is likely explained by the ligament and bone deficiencies resulting from prior multiligament trauma and surgery. Increased prosthetic constraint in knee arthroplasty imparts greater forces on the implants, theoretically contributing to component loosening and failure [5, 8, 31, 47]. However, some studies have reported excellent survivorship of varus-valgus constrained implants in primary and revision TKAs at intermediate followup [1, 18, 19, 30, 41]. The long-term survivorship of these implants is unknown and none of these studies exclusively involved patients with posttraumatic arthritis or prior ligamentous surgery. Not surprisingly, six of eight patients in the multiligament cohort undergoing revision arthroplasty required constrained component designs (four received varus-valgus constrained and two received hinged prostheses). A hinged prosthesis at revision arthroplasty was used in two patients secondary to severe bone loss and severe ligamentous deficiency. Surgeons can be better equipped with this knowledge at the time of primary (or revision) TKA to address intraoperative knee instability by using prostheses with increased levels of constraint.

Complications were far more common among patients undergoing TKA after multiligament knee surgery than among patients undergoing TKA for osteoarthritis. These findings are dissimilar from what has been reported after TKA in patients with a history of ACL reconstruction. Hoxie et al. [13] found no deleterious effects of prior ACL reconstruction on TKA regarding infection, knee motion, or revision arthroplasty. A majority of the revision procedures in the multiligament cohort resulted from TKA instability (5/8). In contrast, Sharkey et al. [49] found that instability accounted for only $8 \%$ of all revision TKAs. Ligamentous insufficiency resulting in TKA instability in our patients was likely attributable to prior multiligament injury, regardless of prior ligament reconstruction or repair. 
We discovered an increased rate of infection in the multiligament cohort compared with the osteoarthritis cohort. Previous studies have shown a higher risk of infection in patients undergoing TKA after prior knee surgery $[2,38]$ or posttraumatic arthritis $[14,34]$. The causality behind our findings is likely multifactorial including retained hardware, poor soft tissue integrity, and increased surgical dissection because of prior surgery. Patients with multiligament injuries are also at increased risk for arthrofibrosis as a result of prior trauma and surgery [28, 29, 44, 54]. While nearly $10 \%$ of patients in the multiligament cohort underwent manipulation under anesthesia for postoperative stiffness, there was no difference in comparison to the matched controls. A potential explanation for this is that the study patients were a young motivated patient population (mean age, 53 years) that was better able to achieve rehabilitation and obtain postoperative knee motion. Several studies have examined knee stiffness after multiligamentous injuries and/or traumatic knee dislocation. Noyes et al. [36] reported a $23 \%$ incidence of knee stiffness in patients undergoing ACL reconstruction and medial collateral ligament repair. Cook et al. [7] reported a $15 \%$ incidence of manipulation under anesthesia and/or arthroscopic lysis of adhesions after multiligament knee surgery. Other studies have shown even higher rates of knee stiffness in patients after traumatic knee dislocations $(30 \%-57 \%)[36,48,50]$.

Interestingly, despite these complications, KSS and improvement in KSS were not different between our study groups. Similarities between the two study populations as a result of matching criteria likely played a role in this outcome. In addition, loss to followup and incomplete data in both study groups introduced the possibility of type II error. Delta KSS and KSS-F scores were tabulated using the most recent followup score available (after TKA or postrevision TKA). Assuming that patients who underwent revision surgery were likely to have lower scores at followup; the higher rate of revision TKA in the multiligament cohort may have negatively influenced the KSS and KSS-F values in this cohort, thus hindering our ability to find a difference between the two groups. Another study examining TKA after ACL reconstruction also did not show a difference in postoperative knee scores compared with those of patients without prior knee surgery [13].

Patients undergoing TKA who have a history of multiligament surgery can expect decreased implant survival, increased risk of revision and reoperation, increased use of prosthesis constraint at index arthroplasty, and a higher rate of infection compared with patients undergoing TKA for osteoarthritis. These findings highlight the importance of clinicians to closely follow this high risk patient population at short- and long-term followup. Additionally, with this knowledge, surgeons might wish to be prepared to use a TKA prosthesis design with increased constraint at the primary TKA. Further research is necessary to determine if a particular multiligamentous surgical technique can prevent posttraumatic arthritis and complications.

Acknowledgments We thank Patrick Reardon MD, Matthew Houdek MD, Youlonda Loechler, and Karen Fasbender from the Department of Orthopaedic Surgery at the Mayo Clinic (Rochester, $\mathrm{MN}$ ) for their help with data collection and submission of this manuscript.

\section{References}

1. Anderson JA, Baldini A, MacDonald JH, Pellicci PM, Sculco TP. Primary constrained condylar knee arthroplasty without stem extensions for the valgus knee. Clin Orthop Relat Res. 2006;442:199-203.

2. Berbari EF, Hanssen AD, Duffy MC, Steckelberg JM, Ilstrup DM, Harmsen WS, Osmon DR. Risk factors for prosthetic joint infection: case-control study. Clin Infect Dis. 1998;27:12471254.

3. Borchers JR, Kaeding CC, Pedroza AD, Huston LJ, Spindler KP, Wright RW; MOON Consortium and the MARS Group. Intraarticular findings in primary and revision anterior cruciate ligament reconstruction surgery: a comparison of the MOON and MARS study groups. Am J Sports Med. 2011;39:1889-1893.

4. Brophy RH, Gray BL, Nunley RM, Barrack RL, Clohisy JC. Total knee arthroplasty after previous knee surgery: expected interval and the effect on patient age. J Bone Joint Surg Am. 2014;96:801-805.

5. Callaghan JJ, O'Rourke MR, Liu SS. The role of implant constraint in revision total knee arthroplasty: not too little, not too much. J Arthroplasty, 2005; 20(4 suppl 2):41-43.

6. Cohen M, Amaro JT, Ejnisman B, Carvalho RT, Nakano KK, Peccin MS, Teixeira R, Laurino CF, Abdalla RJ. Anterior cruciate ligament reconstruction after 10 to 15 years: association between meniscectomy and osteoarthrosis. Arthroscopy. 2007;23:629-634.

7. Cook S, Ridley TJ, McCarthy MA, Gao Y, Wolf BR, Amendola A, Bollier MJ. Surgical treatment of multiligament knee injuries. Knee Surg Sports Traumatol Arthrosc. 2015;23:2983-2991.

8. Donaldson WF 3rd, Sculco TP, Insall JN, Ranawat CS. Total condylar III knee prosthesis: long-term follow-up study. Clin Orthop Relat Res. 1988;226:21-28.

9. Dwyer T, Marx RG, Whelan D. Outcomes of treatment of multiple ligament knee injuries. J Knee Surg. 2012;25:317-326.

10. Engebretsen L, Risberg MA, Robertson B, Ludvigsen TC, Johansen S. Outcome after knee dislocation: a 2 to 9 year followup of 85 consecutive patients. Knee Surg Sports Traumatol Arthrosc. 2009; 17:1013-1026.

11. Fanelli GC, Orcutt DR, Edson CJ. The multiple-ligament injured knee: evaluation, treatment, and results. Arthroscopy. 2005; 21:471-486.

12. Fanelli GC, Sousa PL, Edson CJ. Long-term followup of surgically treated knee dislocations: stability restored, but arthritis is common. Clin Orthop Relat Res. 2014;472:2712-2717.

13. Hoxie SC, Dobbs RE, Dahm DL, Trousdale RT. Total knee arthroplasty after anterior cruciate ligament reconstruction. $J$ Arthroplasty. 2008;23:1005-1008.

14. Jämsen E, Huhtala H, Puolakka T, Mollanen T. Risk factors for infection after knee arthroplasty: a register-based analysis of 43,149 cases. J Bone Joint Surg Am. 2009;91:38-47. 
15. Kaeding CC, Pedroza AD, Parker RD, Spindler KP, McCarty EC, Andrish JT. Intra-articular findings in the reconstructed multiligament-injured knee. Arthroscopy. 2005;21:424-430.

16. King AH, Krych AJ, Prince MR, Sousa PL, Stuart MJ, Levy BA. Are meniscal tears and articular cartilage injury predictive of inferior patient outcome after surgical reconstruction for the dislocated knee? Knee Surg Sports Traumatol Arthrosc. 2015;23:3008-3011.

17. Krych AJ, Sousa PL, King AH, Engasser WM, Stuart MJ, Levy BA. Meniscal tears and articular cartilage damage in the dislocated knee. Knee Surg Sports Traumatol Arthrosc. 2015; 23:3019-3025.

18. Lachiewicz PF, Soileau ES. Ten-year survival and clinical results of constrained components in primary total knee arthroplasty. $J$ Arthroplasty. 2006;21:803-808.

19. Lachiewicz PF, Soileau ES. Results of a second-generation constrained condylar prosthesis in primary total knee arthroplasty. J Arthroplasty. 2011;26:1228-1231.

20. Le DH, Goodman SB, Maloney WJ, Huddleston JI. Current modes of failure in TKA: infection, instability, and stiffness predominate. Clin Orthop Relat Res. 2014;472:2197-2200.

21. Leroux T, Ogilvie-Harris D, Dwyer T, Chahal J, Gandhi R, Mahomed N, Wasserstein D. The risk of knee arthroplasty following cruciate ligament reconstruction: a population-based matched cohort study. J Bone Joint Surg Am. 2014;96:2-10.

22. Levy BA, Dajani KA, Morgan JA, Shah JP, Dahm DL, Stuart MJ. Repair versus reconstruction of the fibular collateral ligament and posterolateral corner in the multiligament-injured knee. Am J Sports Med. 2010;38:804-809.

23. Levy BA, Dajani KA, Whelan DB, Stannard JP, Fanelli GC, Stuart MJ, Boyd JL, MacDonald PA, Marx RG. Decision making in the multiligament-injured knee: an evidence-based systematic review. Arthroscopy. 2009;25:430-438.

24. Levy BA, Fanelli GC, Whelan DB, Stannard JP, MacDonald PA, Boyd JL, Marx RG, Stuart MJ; Knee Dislocation Study Group. Controversies in the treatment of knee dislocations and multiligament reconstruction. $J$ Am Acad Orthop Surg. 2009;17:197-206.

25. Li RT, Lorenz S, Xu Y, Harner CD, Fu FH, Irrgang JJ. Predictors of radiographic knee osteoarthritis after anterior cruciate ligament reconstruction. Am J Sports Med. 2011;39:2595-2603.

26. Lohmander LS, Englund PM, Dahl LL, Roos EM. The long-term consequence of anterior cruciate ligament and meniscus injuries: osteoarthritis. Am J Sports Med. 2007;35:1756-1769.

27. Louboutin H, Debarge R, Richou J, Selmi TA, Donell ST, Neyret P, Dubrana F. Osteoarthritis in patients with anterior cruciate ligament rupture: a review of risk factors. Knee. 2009;16:239244.

28. Lowenstein JE, Levine WN. Complications of surgery for multiligament injuries of the knee. Sports Med Arthroscopy Rev. 2004;12:202-208.

29. Magit D, Wolff A, Sutton K, Medvecky MJ. Arthrofibrosis of the knee. J Am Acad Orthop Surg. 2007;15:682-694.

30. Maynard LM, Sauber TJ, Kostopoulos VK, Lavigne GS, Sewecke JJ, Sotereanos NG. Survival of primary condylar-constrained total knee arthroplasty at a minimum of 7 years. J Arthroplasty. 2014;29:1197-1201.

31. McAuley JP, Engh GA. Constraint in total knee arthroplasty: when and what? J Arthroplasty. 2003;18(3 suppl 1):51-54.

32. Meding JB, Wing JT, Ritter MA. Does high tibial osteotomy affect the success or survival of a total knee replacement? Clin Orthop Relat Res. 2011;469:1991-1994.

33. Meunier A, Odensten M, Good L. Long-term results after primary repair or non-surgical treatment of anterior cruciate ligament rupture: a randomized study with a 15 -year follow-up. Scand $J$ Med Sci Sports. 2007;17:230-237.
34. Namba RS, Inacio MC, Paxton EW. Risk factors associated with deep surgical site infections after primary total knee arthroplasty: an analysis of 56,216 knees. J Bone Joint Surg Am. 2013;95:775782 .

35. Neyret P, Donell ST, Dejour H. Results of partial meniscectomy related to the state of the anterior cruciate ligament: review at 20 to 35 years. J Bone Joint Surg Br. 1993;75:36-40.

36. Noyes FR, Mangine RE, Barber SD. The early treatment of motion complications after reconstruction of the anterior cruciate ligament. Clin Orthop Relat Res. 1992;277:217-228.

37. Oiestad BE, Engebretsen L, Storheim K, Risberg MA. Knee osteoarthritis after anterior cruciate ligament injury: a systematic review. Am J Sports Med. 2009;37:1434-1443.

38. Peersman G, Laskin R, Davis J, Peterson M. Infection in total knee replacement: a retrospective review of 6489 total knee replacements. Clin Orthop Relat Res. 2001;392: 15-23.

39. Pernin J, Verdonk P, Si Selmi TA, Massin P, Neyret P. Long-term follow-up of 24.5 years after intra-articular anterior cruciate ligament reconstruction with lateral extra-articular augmentation. Am J Sports Med. 2010;38:1094-1102.

40. Peskun CJ, Levy BA, Fanelli GC, Stannard JP, Stuart MJ, MacDonald PB, Marx RG, Boyd JL, Whelan DB. Diagnosis and management of knee dislocations. Phys Sportsmed. 2010;38:101111.

41. Peters CL, Hennessey R, Barden RM, Galante JO, Rosenberg AG. Revision total knee arthroplasty with a cemented posterior-stabilized or constrained condylar prosthesis: a minimum 3-year and average 5-year follow-up study. J Arthroplasty. 1997;12:896-903.

42. Piedade SR, Pinaroli A, Servien E, Neyret P. Is previous knee arthroscopy related to worse results in primary total knee arthroplasty? Knee Surg Sports Traumatol Arthrosc. 2009; 17:328-333.

43. Piedade SR, Pinaroli A, Servien E, Neyret P. TKA outcomes after prior bone and soft tissue knee surgery. Knee Surg Sports Traumatol Arthrosc. 2013;21:2737-2743.

44. Richmond JC. Complications associated with the treatment of multiligament-injured/dislocated knee. Sports Med Arthrosc Rev. 2001;9:255-260.

45. Richter M, Bosch U, Wippermann B, Hofmann A, Krettek C. Comparison of surgical repair or reconstruction of the cruciate ligaments versus nonsurgical treatment in patients with traumatic knee dislocations. Am J Sports Med. 2002;30: 718-727.

46. Saleh KJ, Sherman P, Katkin P, Windsor R, Haas S, Laskin R, Sculco T. Total knee arthroplasty after open reduction and internal fixation of fractures of the tibial plateau: a minimum fiveyear follow-up study. J Bone Joint Surg Am. 2001; 83:11441148.

47. Sculco TP. The role of constraint in total knee arthroplasty. $J$ Arthroplasty. 2006;21(4 suppl 1):54-56.

48. Shapiro MS, Freedman EL. Allograft reconstruction of the anterior and posterior cruciate ligaments after traumatic knee dislocation. Am J Sports Med. 1995;23:580-587.

49. Sharkey PF, Lichstein PM, Shen C, Tokarski AT, Parvizi J. Why are total knee arthroplasties failing today: has anything changed after 10 years? J Arthroplasty.2014;29:1774-1778.

50. Sisto DJ, Warren RF. Complete knee dislocation: a follow-up study of operative treatment. Clin Orthop Relat Res. 1985;198:94-101.

51. Stannard JP. The posterolateral corner of the knee: repair versus reconstruction. Am J Sports Med. 2005;33:881-888.

52. Stannard JP. Medial and posteromedial instability of the knee: evaluation, treatment, and results. Sports Med Arthrosc. 2010;18:263-268. 
53. Stannard JP, Stannard JT, Cook JL. Repair or reconstruction in acute posterolateral instability of the knee: decision making and surgical technique introduction. J Knee Surg. 2015;28;450-454.

54. Weiss NG, Parvizi J, Hanssen AD, Trousdale RT, Lewallen DG. Total knee arthroplasty in post-traumatic arthrosis of the knee. $J$ Arthroplasty. 2003;18(3 suppl 1):23-26.
55. Werier J, Keating J, Meek RN. Complete dislocation of the knee: the long-term results of ligamentous reconstruction. Knee. 1998;5:255-260.

56. Wilson SM, Mehta N, Do HT, Ghomrawi H, Lyman S, Marx RG. Epidemiology of multiligament knee reconstruction. Clin Orthop Relat Res. 2014;472:2603-2608. 